\title{
Some views on the mapping of erosion of coated composites in tidal turbine simulated conditions
}

\author{
Ghulam Rasool and Margaret M. Stack \\ Department of Mechanical \& Aerospace Engineering, University of Strathclyde \\ James Weir Building, 75 Montrose Street
}

Glasgow, UK

Ghulam.rasool@strath.ac.uk

Margaret.Stack@strath.ac.uk

\begin{abstract}
This paper presents a study of the erosion resistance of coated and uncoated polymer matrix composites for tidal turbine conditions. It focuses on the development of comparative erosive wear mode and mechanism maps for such materials. In our earlier work, i.e. testing of glass fibre reinforced polymer composites for tribological applications in marine simulated conditions, several erosion related issues were highlighted. The combined effects of the $\mathrm{NaCl}$ solution and sand enhanced the erosive wear dramatically of the uncoated specimens. In order to address those issues, an erosion resistant polymeric coating was applied to the composite and tested in marine simulated conditions with an extended range of sand particle size. The test results of the uncoated and coated composite have been compared in this research by erosive wear mode and mechanism maps techniques. These maps reveal that the coating has enhanced the erosion resistance. These findings provide significant progress towards materials selection approaches to manufacture of tidal turbine blades.
\end{abstract}

Keywords: Tidal turbine blade, leading edge erosion, polymeric composite and coatings, erosion maps 


\section{Introduction}

Decreasing amounts of fossil fuel reserves and increasing awareness of environmental issues have forced a significant increase in research of renewable energy sources, such as wind, marine, solar, and biomass electricity generation technologies. Of these technologies, marine energy has significant advantages compared to other available renewable energy resources. Marine tidal energy is extremely predictable and can be developed without the destruction of environmental infrastructure [1]. Furthermore, the higher density $(x$ 784) of seawater (compared to air) means that a tidal turbine can produce much higher power than the equivalent diameter wind turbine operating at similar impact velocities [2].

Contrary to the above-mentioned advantages, there are still several challenges facing the tidal power industry before commercialization and full adoption of such technologies on a global scale. These can be summarized as technical, design, materials, reliability, cost, operation and maintenance issues. In particular, the capital cost and rotor blade material reliability of the tidal turbine are major barriers to harnessing this technology [3]. The tidal turbine blade is an important part of this device which converts tidal into mechanical energy to rotate an electric generator. The materials of the rotor blade must be erosion and corrosion resistant in salt water, have favourable antibiofouling properties, and sufficiently robust to withstand the aggressive marine environment. [4].

Polymer composites are being used in the manufacturing of the wind turbine blades. Fibre reinforced polymer composites have favourable mechanical properties and higher strength to density ratio and corrosion resistant qualities. Similar to a wind turbine, fibre reinforced polymer composites can be used as tidal turbine blade materials following appropriate surface treatment.[5]. To date, there is no optimum design and selection of a suitable material for a tidal turbine blade. Tribological issues, such as rotor blade erosion, cavitation, and effects of combined impact of solid particle and seawater need to be addressed to optimize materials performance [6].

In our previous research work, a commercially available composite G10 epoxy glass laminate was tested in a tidal turbine laboratory simulated conditions for leading edge erosion evaluation of a rotor blade in seawater conditions containing particles. The test results showed various tribological issues, such as matrix cutting, reinforcement fracture, mass 
gain, salt solution interaction, swelling, fibre and matrix de-bonding, and reinforcement degradation resulting in mass gain and erosion of the exposed surface [7]. Similar erosive wear behaviour of reinforced composite materials was found by Zahavi et al. in their study of such materials [8].

The literature shows that a glass fibre reinforced unsaturated polyester laminate gains weight by moisture absorption when immersed in saltwater for different long periods. The mechanical properties, such as bending and tensile strength are decreased when it is kept immersed in salt water for a longer period [9]. Moreover, G10 epoxy glass laminate has shown mass gain by the diffusion of the salt solution. Increases in the overall mass of the rotor blades can raise operational issues such as unbalancing of the tidal turbine [10].

Furthermore, an increase in the mass of the rotor blade by diffusion can play a significant role in the tribological behaviour of the polymer composite. At the start, the moisture passes through a fibre-matrix interface through the pores and void in the matrix by a diffusion process. This results in swelling of the matrix, a reduction in adhesion between the reinforcement and the resin, and de-bonding of reinforcement fibre and matrix at the interface [7]. These factors accelerate the mass loss rate of the material by erosion. The absorption of moisture may result in extraction of soluble elements of the composite material in a salt solution. The net mass variation is equal to the difference of mass gain by diffusion and mass loss by extraction processes $[9,10,11,12]$.

Similar to a wind turbine blade, the leading edge erosion of a tidal turbine blade results in roughened surfaces. A damaged exposed surface of the rotor blade tends to raise different problems, such as rapid moisture absorption and accelerated mass gain, increase in Reynolds number, which leads to an increase in drag and decrease in lift, resulting in an unbalanced blade operation. These factors compromise the efficiency, availability, and reliability by increasing the rate of failures, maintenance and operational cost, and downtimes for the tidal turbines $[13,14,15,16]$. For sustainable energy resources, the marine renewable energy (MRE) industry needs to ensure that MRE devices must not raise any environmental issue and are maintenance free to complete their life cycles [17].

To overcome the above mentioned tribological and diffusion-related issues to enhance the performance and life cycle of fibre-reinforced composites in the marine environment, an 
erosion resistant polymeric coating was applied to the $\mathrm{G} 10$ epoxy glass laminate substrate. Both the uncoated and coated specimens were tested in a scaled laboratory simulated tidal turbine erosion rig. Mass change and SEM analysis were carried out for both materials. On the basis of test results, erosion mode and mechanism maps have been constructed and presented in this paper. These erosion maps show that erosion resistant polymeric coatings functioned as a barrier between seawater and the substrate along with an enhancing erosion resistance of the substrate in the marine environment. The increase in erosion resistance of this fibre reinforced composite by the application of coatings is an important development towards the selection and use of advanced materials for the manufacturing of the tidal turbine blades. Future work will involve exploring and testing of further advanced materials and hybrid composite coatings for such applications.

\section{Experimental}

\subsection{Material}

A commercially available composite G10 epoxy glass laminate was used for this study as an uncoated specimen and as a substrate for the polymer coatings. This composite has favourable mechanical properties such as high strength, high stiffness, and high creep resistance. This material is being used in several industries, such as aerospace, medical, and power generation under seawater applications. It is comprised of epoxy resin with continuous glass fibre cloth reinforcement. Moreover, this thermosetting material has low moisture absorption and corrosion resistance in both onshore and offshore environments. The size of specimens was $60 \times 25 \times 6 \mathrm{~mm}$ by length, breadth, and thickness. Figure 1(a) shows the pre-test SEM image of the uncoated specimen. The material properties are as below:

Table 1. G10 epoxy glass laminate properties

\begin{tabular}{|l|l|}
\hline Flexural strength $(\mathrm{MPa})=482$ & Tensile strength $(\mathrm{MPa})=320$ \\
\hline Shear strength $(\mathrm{MPa})=131$ & Charpy impact strength $\left(\mathrm{kJ} \mathrm{m}^{-2}\right) 65$ \\
\hline Density $\left(\mathrm{g} \mathrm{cm}^{-3}\right)=2$ & Specific gravity $=2$ \\
\hline Water absorption $\left(\mathrm{mg} \mathrm{m}^{-2}\right)=8$ & Hardness, Rockwell $(\mathrm{m}$-scale $)=110$ \\
\hline Body colour $=$ Green & Standard finish = Satin/Glossy \\
\hline
\end{tabular}



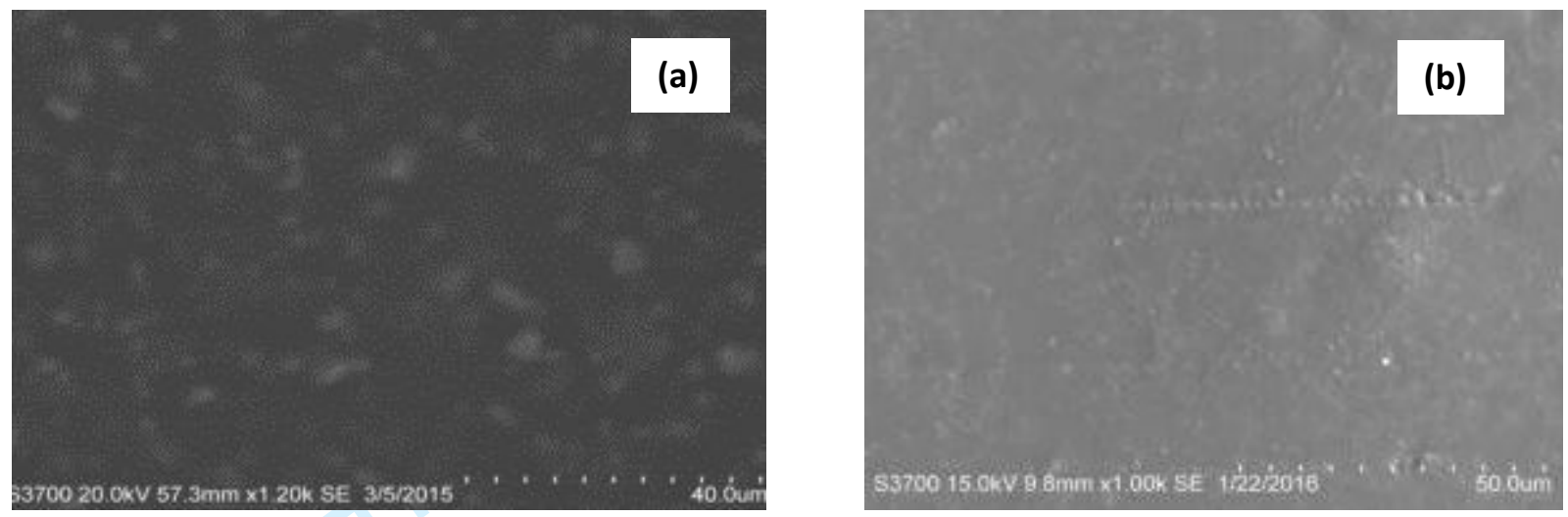

Figure 1: (a) SEM image of the uncoated specimen, and (b) SEM image of the coated specimen.

\subsection{Coating}

Erosion and corrosion resistant polymeric coating were tested in this research work. This coating process is a rapid and cold cure. The coatings were supplied and generated by Belzona Polymerics Ltd. Using on our samples above as substrates. This polymeric coating consists of two components, the base component is an Epoxy Phenol Novolac Resin, and the other solidifier component is a Polyalkene Amine. The combination ratio for the base to solidifier component for the coating is; 2.2 parts base: 1 part solidifier (by weight). These components are the uncured Epoxy resin, which when mixed, begins the curing process to form a polymeric coating. The chemical composition of base and solidifier is shown separately in Table 2. As a preparation process, the specimen surfaces were roughened to $75 \mathrm{Ra}(\mu \mathrm{m})$ with $100 \mu \mathrm{m}$ chilled iron grit and cleaned with a cleaner agent. After preparation of samples, the coating was applied by a hand short bristle brush. The average thickness of the coatings was $0.5 \mathrm{~mm}$ [18]. Fig. 1 (b) shows the pre-test SEM image of the coated specimen. The mechanical strengths and other material properties are shown in Table 3.

Table 2. Chemical composition of base component and solidifier component

\begin{tabular}{|l|l|}
\hline Base component & Quantity \\
\hline Epoxy Phenol Novolac Resin & $100 \%$ \\
\hline Solidifier component (Mixture) composition & Quantity \\
\hline
\end{tabular}




\begin{tabular}{|l|l|}
\hline Polyalkylene Amine & $55-60 \%$ \\
\hline Phenol, Styrenated & $25-30 \%$ \\
\hline 2-Piperazine-1-Yl Ethylamine & $10 \%$ \\
\hline $\begin{array}{l}\text { Formaldehyde Polymer with 1.3 } \\
\text { Benzenedimethanamine and Phenol }\end{array}$ & $1-5 \%$ \\
\hline
\end{tabular}

Table 3. Coating properties

\begin{tabular}{|l|l|}
\hline Flexural strength $(\mathrm{MPa})=43.1$ & Flexural Modulus $(\mathrm{MPa})=2037.4$ \\
\hline Mandrel Flexibility $=$ Pass at $2.5^{\circ} /$ pipe dia. & Tensile shear strength $(\mathrm{MPa})=26.9$ \\
\hline $\begin{array}{l}\text { Pull off adhesion (Posi Test Dolly Pull off) } \\
(\mathrm{MPa})=22.1\end{array}$ & $\begin{array}{l}\text { Taber Abrasion: Wet }(\mathrm{H} 10 \text { Wheels })=46 \mathrm{~mm}^{3} \\
\text { Dry }(\mathrm{CS} 17 \text { Wheels })=13 \mathrm{~mm}^{3} \text { loss per } 1000 \\
\text { cycles }\end{array}$ \\
\hline Elongation $=1.12 \%$ & Young's Modulus $(\mathrm{MPa})=1963.6$ \\
\hline Compressive yield strength $(\mathrm{MPa})=39.8$ & Compressive Modulus $(\mathrm{MPa})=784.6$ \\
\hline Density $\left(\mathrm{g} \mathrm{cm}^{-3}\right)=1.14$ & Hardness, Shore $\mathrm{D}=77$ \\
\hline Relative density $=1.14$ & Water absorption $\left(\mathrm{mg} \mathrm{m} \mathrm{m}^{-2}\right)=\mathrm{Nil}$ \\
\hline
\end{tabular}

\subsection{Test slurries and test conditions}

Two types of saltwater (sea water analogue) plus sand slurry were used for the testing of the uncoated and coated polymeric composite substrate. Sand was divided into two groups on the basis of particle size as Sand 'A' $(200 \pm 50 \mu \mathrm{m})$ and Sand 'B' (425 $\pm 175 \mu \mathrm{m})$; the shape of sand particles was sub-angular. The salinity of the slurry was $3.5 \%$ salt and sand concentration was $3 \%$ of the mass of the water. The test was run in room ambient conditions for 2 hours at the rotor blade tip velocity of $3 \mathrm{~ms}^{-1}$ of the rotor blade. The slurries were changed after half an hour of the test run during each experiment to reduce the effects of sand particle shape attrition. The range of angles of attack tested was $0^{\circ}$ to $90^{\circ}$ with an increment of $15^{\circ}$.

The angle of attack plays an important role in the leading edge erosion of the tidal turbine blades. Figure 2 shows the schematic of an incident of an angle of attack at the leading edge of the rotor blade. The leading edge is located at the forward intersection point of the upper and lower surface of the hydrofoil of the rotor blade. A straight chord line passes through 
the leading edge and trailing edge of the hydrofoil. The angle of attack ' $\alpha$ ' shown in Fig. 2, is between the relative velocity vector and the chord line. An angle of attack depends on the shape and profile of the hydrofoil of a rotor blade. A rotor blade is designed to extract maximum power from the marine tide flow stream on the basis of lift force; drag force and camber of the hydrofoil.

The range of angles of attack was selected in view of the numerical analysis and experimental results available in the literature for the operating conditions of the rotor blades. Muratoglu et al. [23] had carried out a performance basis numerical analysis by using JavaFoil Code on the already developed hydrokinetic turbine blade with respect to angles of attack. They studied lift, drag and pressure coefficients of different hydrofoils, such as NACA, NREL, and RIS $\phi$ hydrofoils. On the basis of comparison of JavaFoil, XFOIL, EllipSys2D, and experimental results they found that an angle of attack $15^{\circ}$ had the highest value of the coefficient of lift [23].

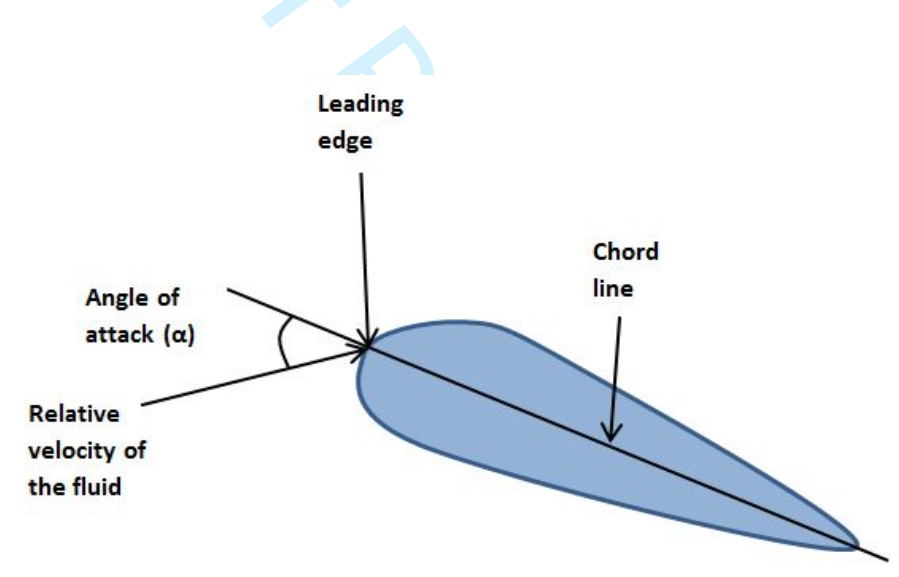

Figure 2: The angle of attack on the leading edge of the hydrofoil [16].

The lift coefficient has a small value at the lower angle of attacks, which increases with the increase in angle of attack in a linear manner until it reaches the highest value. Then it decreases abruptly and the hydrofoil stalls as the angle of attack increases. On the other hand, a coefficient of drag increases with an increase in the angle of attacks [16]. In a technical review, Fraenkel [24] has stated that hydrofoils stalling results in a sudden increase in drag and decrease in efficiency. Therefore the rotor blades are designed to operate just before the stalling point. He concluded that a good shaped cambered hydrofoil with a cylindrical leading edge is more efficient and operates at higher angles of attack as 
compared to other unfavourable shaped ones. Moreover, there is a need to twist tidal turbine blades to operate at different angles of attack at various radii [24]. Like a wind turbine blade, there are three main phases for the erosion of the rotor blade as upper core breach, lower core breach and leading edge breach. Therefore the erosion may occur on any exposed surface of the rotor blade [51]. That is why this experimental work was carried out for a range of angles of attack $0^{\circ}$ to $90^{\circ}$ to establish a database for the leading edge erosion of the tidal turbine blade.

Moreover, in view of the above mentioned experimental conditions, the tip speed of the rotor was optimised to get a steady and an undisturbed flow of the slurry in the test chamber to investigate the leading edge erosion due to the solid particle in a marine simulated environment. The cavitation damage was not considered at this stage because the required vapour pressure could not be reached to generate bubbles in the slurries. Moreover, the difference between the root and tip speed was also ignored in this paper. The solid particles were treated at rest and the tip speed was considered as the speed of impingement [25].

\subsection{Test Apparatus}

A slurry pot test rig was used for this experimental work to simulate laboratory conditions of a tidal turbine (the conditions have been scaled down for realistic tidal turbine conditions as identified by CFD modelling and identification of thrust loads on the blade surface) Fig 3 . This rig has two motors, the upper function as a tidal turbine rotor and the bottom maintain the sand slurry in suspension in the test chamber. Two specimens can be tested at the same time at different angles of attacks. A range of angles attack can be tested in this configuration. The four baffles bars in the test chamber are installed to minimise swirling and centrifugal effects. These bars also provide a flow of the slurry in the axial direction. The upper and bottom motors rotate in different directions to ensure even mixing of the sand particles in solution and to maintain an even flow of the slurry in the test chamber [19 - 22]. 

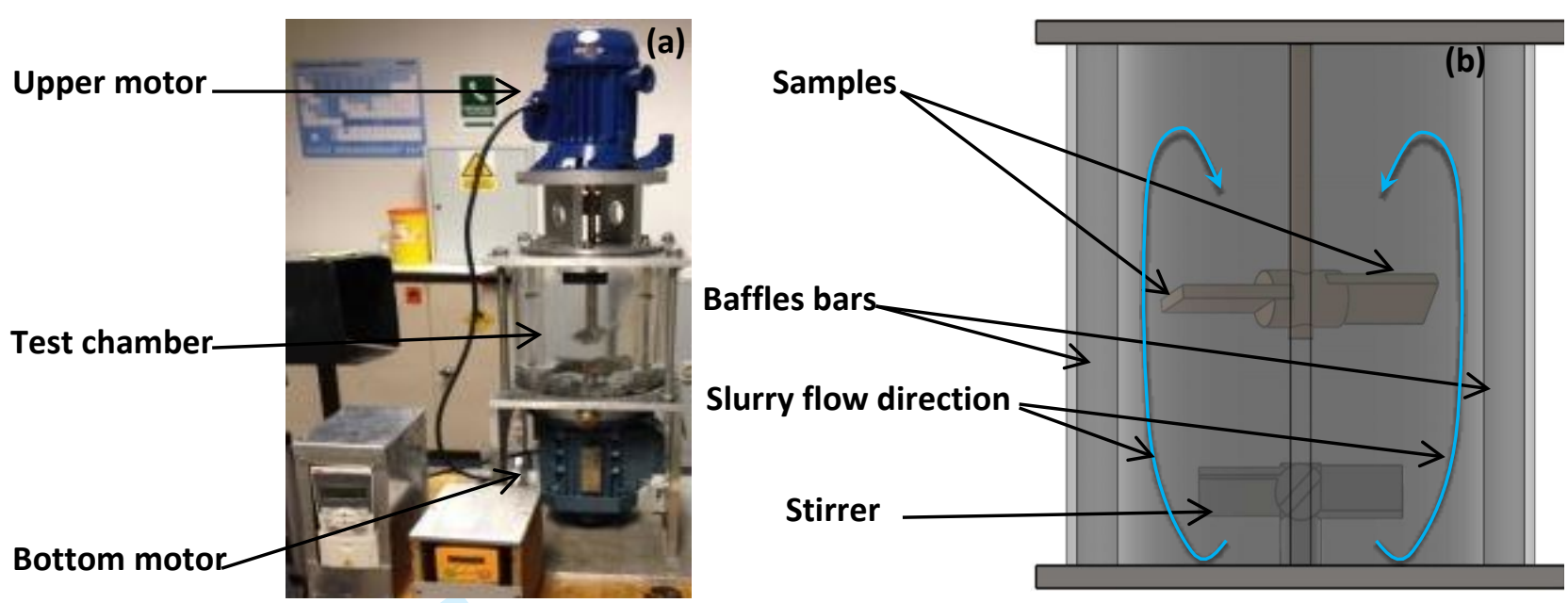

Figure 3: (a) The slurry pot test rig, and (b) the test chamber.

\subsection{Test Methodology}

The test samples were cut into a size of $60 \times 25 \mathrm{~mm}$ from a G10 epoxy glass laminate sheet of $6 \mathrm{~mm}$ thickness. The specimens were cleaned and weighed on an analytical balance prior to and after the test run. The accuracy of this electronic balance was $0.1 \mathrm{mg}$. The front face position of the sample in the specimen holder was taken as an impact angle of the slurry [20]. A vortices free lower impact velocity was chosen for this experimental work [22]. The axial movement of the slurry was confirmed by a high speed camera by analysing the trajectory of the erodent in the test chamber. The impact velocity of the specimen was calibrated for each combination of test slurry and impact angle. The uniform distribution of the erodent in the salt water plus sand slurry was maintained by the stirring process of the bottom motor [21].

Before starting an experiment, the test chamber was filled with slurry and removed any trapped air from the chamber. At the end of the test, each sample was rinsed in pure water and blown with a gentle heat to minimise the effect of water absorption. The mass loss was measured 24 hours later after keeping the specimen at the room temperature. For the erosion comparison of the uncoated and coated substrate, the volume losses were calculated from the densities of both materials. SEM was used to compare the erosion behaviour of the uncoated and coated substrate. 


\section{Test results}

\subsection{Erosive wear}

Figure 4 (a) shows the volume loss of uncoated specimens in salt water + Sand A (200 \pm 50$)$ $\mu \mathrm{m}$ slurry and Figure 4 (b) shows the volume loss of coated specimens in salt water + Sand $B$ $(425 \pm 175) \mu \mathrm{m}$ slurry. With respect to volume loss, both graphs show high wear of the uncoated composite up to the $15^{\circ}$ angle of attack, while, for the coating, high erosion is observed at a $30^{\circ}$ angle of attack. On the other hand, there is low wear prevalent for the uncoated composite at an impact angle of $75^{\circ}$ and for further higher impact angles. For the coated specimen, there is low wear for the range of angles of attack except $30^{\circ}$.
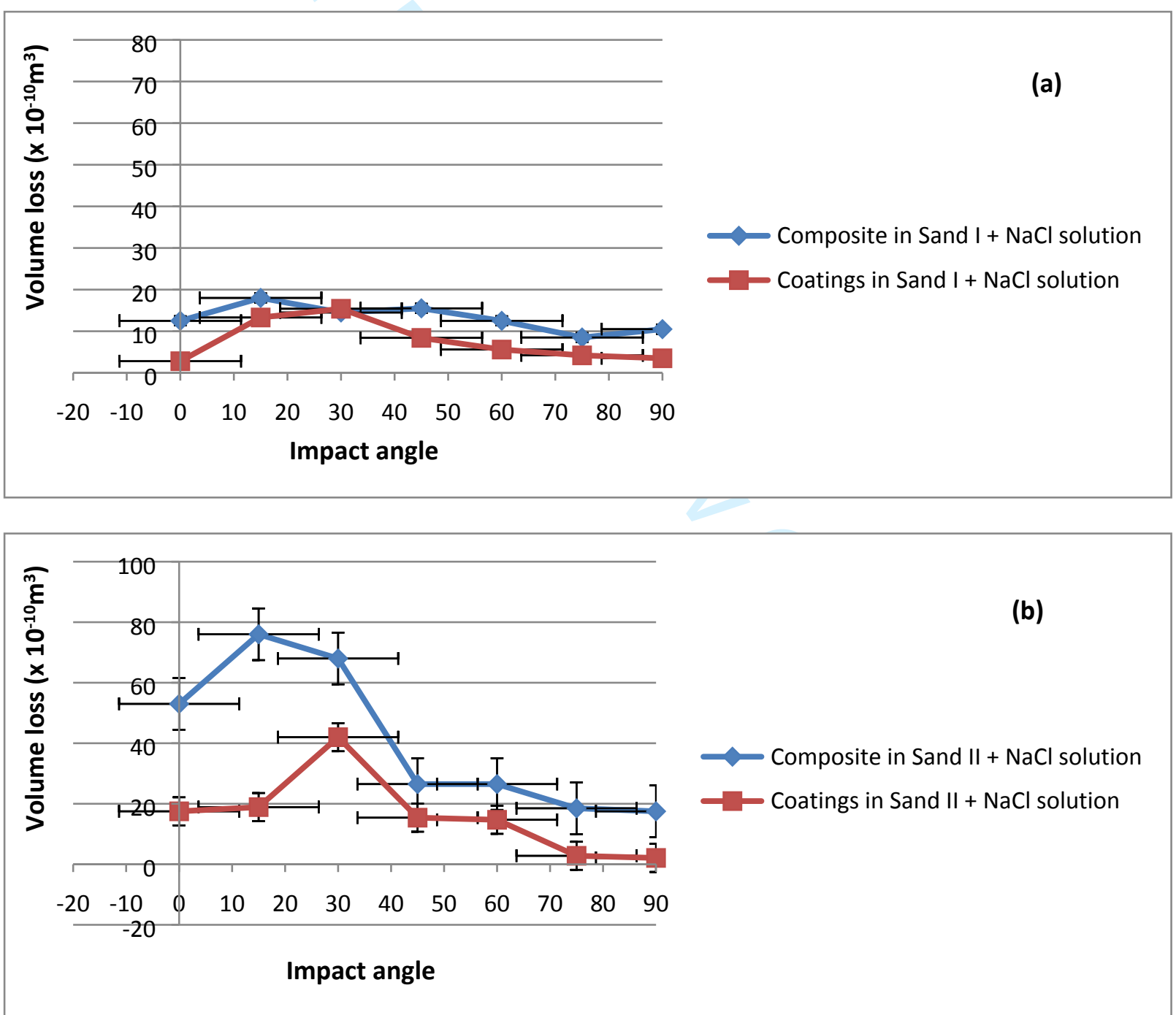

Figure 4: (a) Erosive wear of uncoated and coated specimens, in salt water + Sand A (200 $\pm 50 \mu m)$ slurry, and (b) shows the erosive wear of coated specimens in salt water + Sand B (425 $\pm 175 \mu \mathrm{m})$ slurry. 


\subsection{SEM images of the eroded surfaces}

3.2.1. SEM images of the eroded surfaces in salty water + Sand A $(200 \pm 50 \mu \mathrm{m})$ slurry.

Figure $5(a, b, c, d)$ shows SEM images of the eroded surfaces of the uncoated specimens in salt water + Sand A slurry. These images show the exposed fibres, blisters, and damaged surfaces and fractured fibres, due to the cutting and impact of the erodent. Figure 5 (e, $f, g$, h) shows SEM images of the eroded surfaces of the coated specimens in salt water + Sand A slurry. In these images, the micro-cutting action of the erodent dominates the coated surfaces at $15^{\circ}$ and $30^{\circ}$ angles of attack. Figure 5 (f) shows that there is an increased intensity of cutting at $30^{\circ}$ but the coating was not worn through to the substrate.
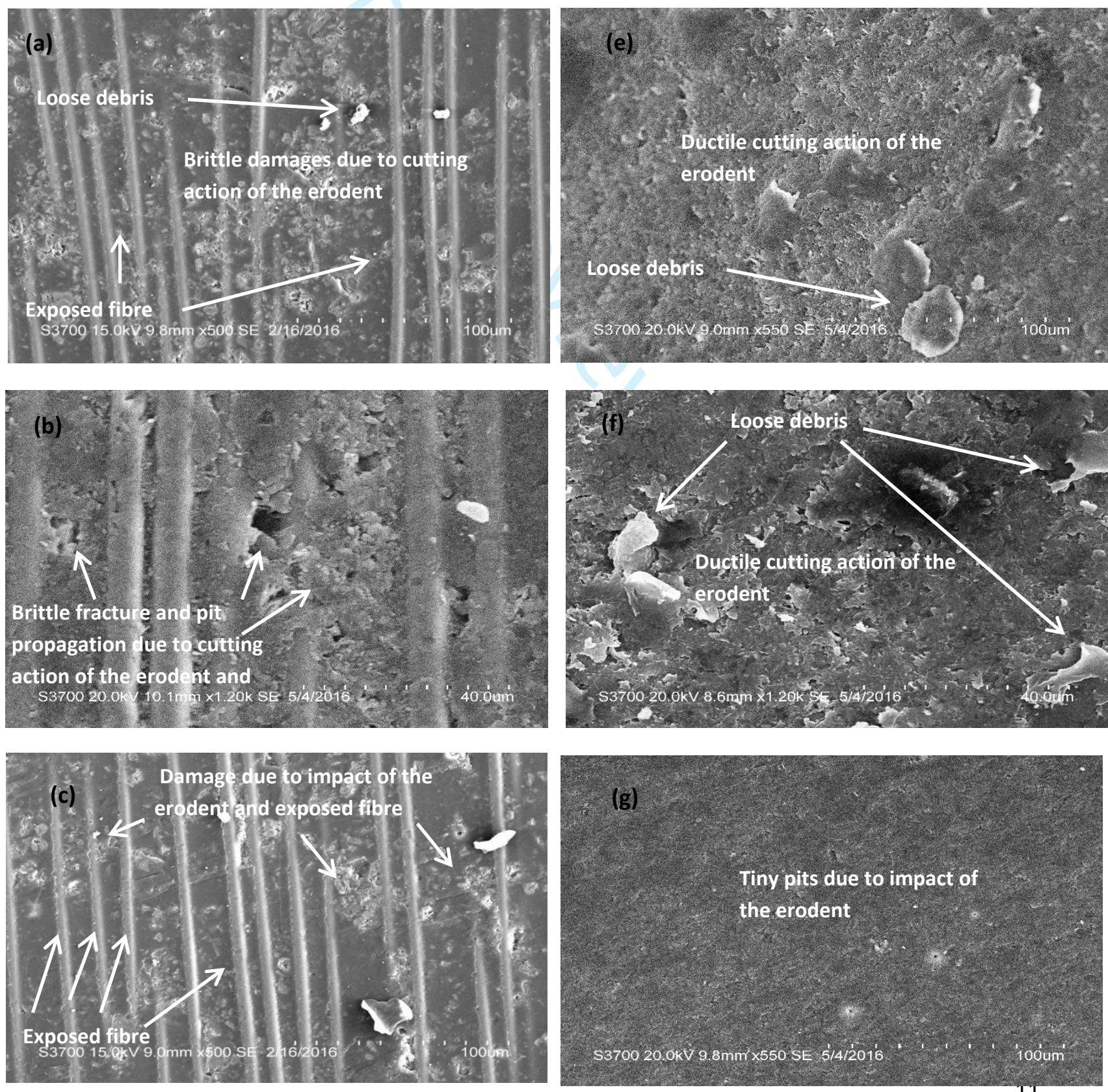

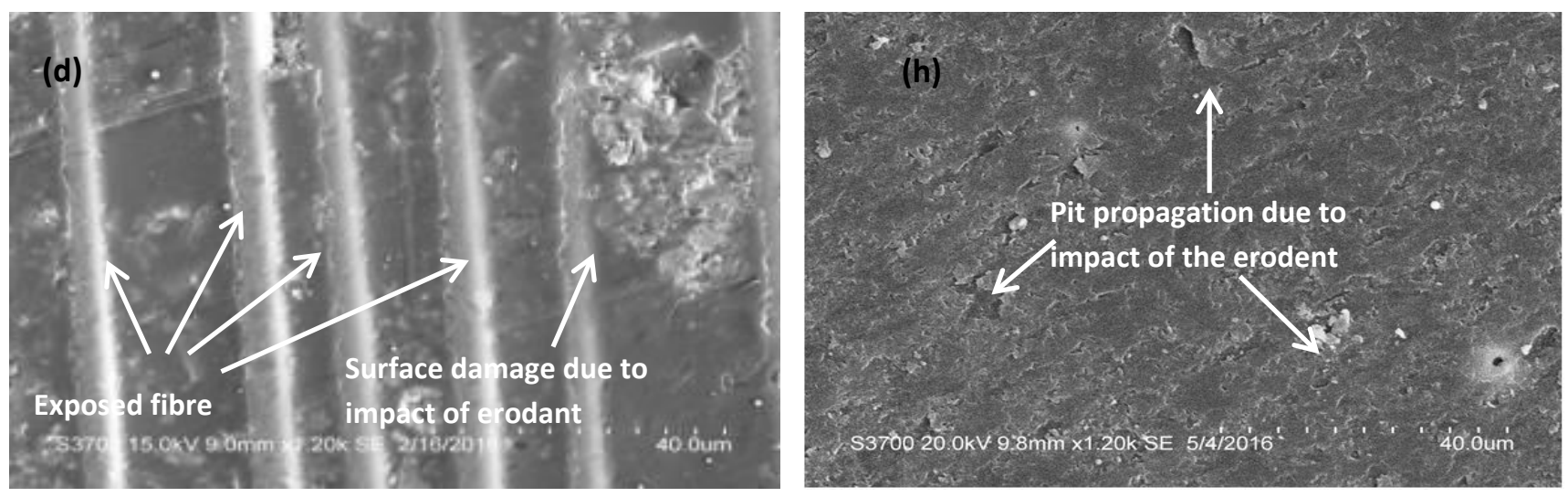

Figure 5: SEM images of the eroded surfaces of the specimens in salt water + Sand A $(200 \pm 50 \mu m)$ slurry, (a) uncoated specimen at angle of attack $15^{\circ}$, (b) uncoated at angle of attack $30^{\circ}$, (c) uncoated at angle of attack $60^{\circ}$, (d) uncoated specimen at angle of attack $75^{\circ}$, (e) coated at angle of attack $15^{\circ},(f)$ coated at angle of attack $30^{\circ},(\mathrm{g})$ coated at angle of attack $60^{\circ}$, and $(\mathrm{h})$ coated at angle of attack $75^{\circ}$.

\subsubsection{SEM images of the eroded surfaces in salt water + Sand B $(425 \pm 175 \mu \mathrm{m})$ slurry.}

Figure $6(a, b, c, d)$ shows SEM images of the eroded surfaces of the uncoated specimens in salt water + Sand B slurry. In these images, large size sand particles have significantly damaged the exposed surfaces. The sharp cutting action of the erodent can be seen at $15^{\circ}$ and $30^{\circ}$ angle of attack Fig. $6(a, b)$. Fracture of the matrix and exposure of fibre could be seen on the exposed surfaces as a result of cutting action of the erodent in these images. At $60^{\circ}$ and $75^{\circ}$ angles of attack, erosive wear Fig. 6 (c, d), results in severe brittle erosive wear of the uncoated composite.

Figure $6(e, f, g, h)$ shows SEM images of the eroded surfaces of the coated specimens in salt water + Sand B slurry. These images show that erosion mechanism progressing in the same manner from $0^{\circ}$ to $90^{\circ}$ angles of attack as that of in the case of the uncoated composite. However, the polymeric coating material showed different erosion behaviour, i.e. "ductile erosion". As it is evident from Fig. 5 (e, f, g, h) and Fig. 6 (e, f, g, h) the coating showed good adhesion to the substrate and did not wear through to the substrate. 

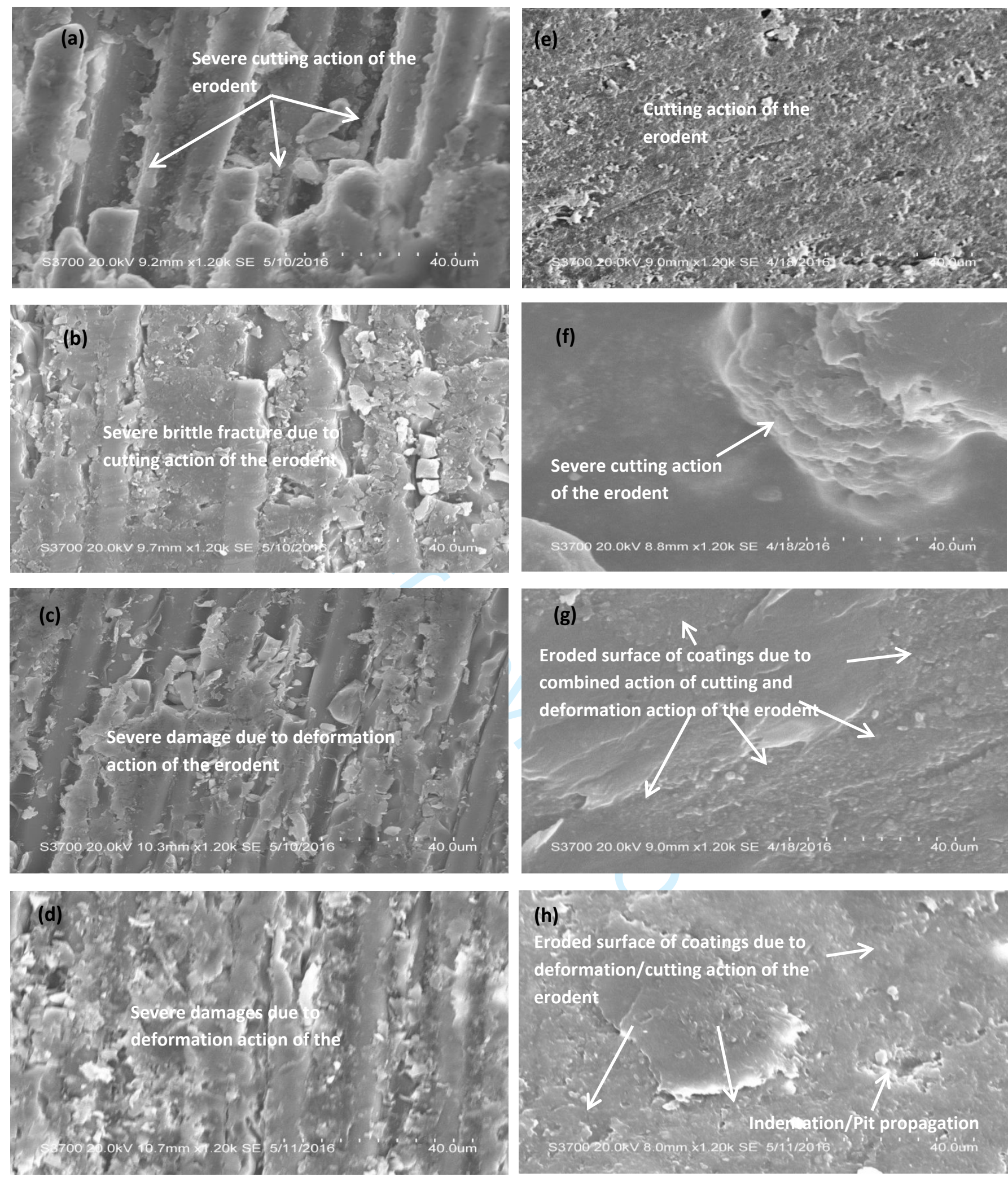

Figure 6: SEM images of the eroded surfaces of the specimens in salt water + Sand B (425 $\pm 175 \mu \mathrm{m})$

slurry, (a) uncoated specimen at angle of attack $15^{\circ}$, (b) uncoated at angle of attack $30^{\circ}$, (c) uncoated at angle of attack $60^{\circ}$, (d) uncoated at angle of attack $75^{\circ},(e)$ coated specimen at angle of 
attack $15^{\circ},(\mathrm{f})$ coated at angle of attack $30^{\circ},(\mathrm{g})$ coated at angle of attack $60^{\circ}$, and $(\mathrm{h})$ coated at angle of attack $75^{\circ}$.

\section{Discussion}

The efficiency of the tidal turbine depends on the performance of the rotor blade. The rotor blade is an important and essential part of the tidal turbine, which extracts kinetic energy from the tide stream. The working concept of the tidal turbine blade is similar to that of the wind turbine blade, but the design and reliability assessment procedure of the wind turbine blade cannot be applied due to several reasons, mainly, the heavy load on the tidal turbine blade due to the higher density of the seawater [26]. There are several technologies and material related factors which compromise the performance and reliability of the rotor blade. One of these factors is the tribological issue, such as leading edge erosion by the impact of the sand particles, erosion by cavitation, and the combined effects of seawater and solid particles on the tidal turbine blade. In this paper, the erosion of the rotor blade due to the impact of the erodent in marine simulated conditions, i.e. salt water plus sand particles, has been investigated. The erosion due to cavitation was ignored because of optimised tip speed and atmospheric pressure, which were not appropriate for reaching a vapour pressure to generate bubbles for cavitation [27].

As a part of the research programme on advanced materials for the renewable devices, a commercially available fibre reinforced polymer composite G10 epoxy glass laminate substrate and coated with a polymer composite erosion resistant coating was tested in a laboratory simulated tidal turbine blade conditions. Test results of the uncoated composite revealed a different erosion behaviour of this composite than that of metal. So-called ductile or brittle erosion behaviours of metals have not been followed by this material Fig. 4 (a) \& (b). The volume loss trend of the substrate shows semi ductile erosion behaviour of this material. The variation of erosive behaviour of the fibre-reinforced composite can be attributed to its material class, mechanical properties, and combined effects of the erodent and seawater $[7,28,29,52]$.

For marine devices, wear of structural materials is a continuous process. The combined effects of solid particle and seawater result in a gradual degradation of materials [7]. Several factors relating to the particle, target material and the environment contribute to the 
degradation of the tidal turbine blades. The effects of these parameters are apparent in Fig. 4. The volume loss of substrate and coatings increased with an increase in sand particle size Fig. 4 (a) \& (b). This increase in erosion rate is in agreement with the research work found in the literature. For solid particle erosion, the volume loss depends on the solid particles, which increases with the increase in size, angularity, and hardness of the erodent. This trend is not always constant at higher concentrations of solid particles. Higher concentrations result in the dissipation of kinetic energy of the solid particle due to mutual collisions, blanketing and limited rotation in the stream $[22,28]$.

By correlating the volume loss of the substrate, Fig. 4 (a) \& (b) with the topographies of the eroded surfaces in Fig. 5 (a, b, c, d) \& Fig. 6 (a, b, c, d), it is apparent that the cutting action of the erodent leads to high volume loss as well as degradation of the exposed surfaces at the lower angles of attack. At the higher impact angles, brittle fracture of the matrix of the uncoated composite dominates in these images for both slurries. In the context of fibre reinforced composite as a tidal turbine blade material, these surface degradation processes can raise several issues, such as leading edge erosion, rapid rate of diffusion and accelerated mass gain and an increase in Reynolds number, which results in a decrease in the lift to drag ratio and reducing the operational efficiency of the turbine blade.

The increase in mass plays a significant role in the degradation of the fibre reinforced composite material. Initially, the moisture diffuses to the fibre-matrix interface through the pores and voids. The absorbed moisture can result in swelling of the matrix, crack initiation, pit propagation, reduction in adhesion between reinforcement and resin, de-bonding of reinforcement fibre and matrix at the interface, and the decrease in mechanical properties. These results are consistent with our previous study for this substrate [7, 11, 27]. The absorbed moisture results in the extraction of soluble matter from the composites into the slurries. The net mass variation will be the difference of mass gain by diffusion and mass loss by the extraction processes $[9,10,11,12]$. All these rotor blade material related factors affect the performance and life cycle the marine tidal turbines $[13,14,15,16]$.

The unique trend of solid particle erosion, i.e. the ductile and brittle material erosion behaviour depends on the properties of the erodent. Erosion of ductile material may peak at a lower angle of attack, while the erosion of nominally brittle one peak at $90^{\circ}$ [31]. 
Furthermore, it is clearly mentioned in the literature that rubber and thermoplastic polymer these observations, the uncoated composite follows "semi ductile" erosion behaviour, while the polymer coating follows "ductile" behaviour of this tribo-system. This is to point out that classical erosion of materials does not hold especially when the particle geometry changes, e.g. from angular to round one; in all, the erosion trend is determined by the properties of the erodent as well as that of target materials [32].

Contrary to the substrate, the use of erosion resistant coating reduces the volume loss Fig. 4. The coated specimens show a peak in volume loss at an angle of attack $30^{\circ}$ in both slurries, which is a ductile erosive behaviour of this material Fig. 4 (a) \& (b). The erosion behaviour of this composite is in agreement with the research work found in the literature $[8,31,32]$. Other work has shown that the peak in volume loss due to erosion depends on the characteristics of the erodent as well [33]. The cutting action of the erodent dominates at lower angles of attack, which results in higher volume loss of the coating at $30^{\circ} \mathrm{Fig}$. 4 , Fig. $5(e, f)$, Fig. $6(e, f),[30]$. The exposed surface topographies show the volume loss is followed by the deformation of the upper layers of the coating, Fig. $5(g, h)$, Fig. $6(g, h)$, [8]. The coating acts as a barrier between saltwater and substrate, remains intact with good adhesion to the substrate and protects the composite from the tribological as well as environmental damage Fig. 5 (e, f, g, h), Fig. 6 (e,f, g, h). Moreover, this polymeric coating remains insoluble as well as non-reactive with saltwater [18, 53, 54]. Despite these developments, there are still erosion issues with this coating, which need to be addressed in order to increase the efficiency of such devices by reducing the drag forces on the rotor blades [47].

\section{Erosive wear maps}

Wear maps are a powerful tool for the investigation of a tribo-system data in a comprehensive manner. These maps provide basic information and a guide to the designers and engineers in the selection of appropriate materials, system operating conditions, prediction of wear behaviour, mechanisms of failures of components and devices for the tribological aspects. These can be categorised as erosion mode maps, erosion-transition maps, erosion-regime maps, and erosion-mechanism maps. Generally, wear-mode, weartransition and wear-regime maps focus on the description of the wear mode, while the wear 
mechanism map details the mechanisms of wear and highlights the regions of wear dominance of a tribo-system. The wear modes and mechanisms transitions boundaries are approximated on these maps. The erosion modes can be termed as low erosion, medium erosion, and severe erosion [34 - 37]. These maps have been constructed for a range of angles of attack $0^{\circ}$ to $90^{\circ}$ to establish a database for the leading edge as well as overall erosion of the tidal turbine blade.

\subsection{Erosion mode maps}

In view of the experimental observations such as volume loss, exposed surface topographies, and literature, empirical wear mode maps have been constructed of uncoated and coated composite substrate to assess the coating performance Fig. 7. These maps can be a useful technique for the investigation and prediction of mode degradation, level of wastage, and potential 'safe' and 'unsafe' operation conditions for a tidal turbine material. The following erosive wear mode regimes (a) very low erosion, (b) low erosion, (c) medium erosion, and (c) severe erosion are defined in view of volume loss, consistent with research literature, and physical observations of the exposed surfaces such as SEM images [34 - 37].

Erosion mode regime boundaries:

a) Very low erosion $\leq\left[\left(5 \times 10^{-10}\right) \mathrm{m}^{3}\right]+$ exposed surface topography

b) $\left[\left(5 \times 10^{-10}\right) \mathrm{m}^{3}\right]<$ Low erosion $\leq\left[\left(12 \times 10^{-10}\right) \mathrm{m}^{3}\right]+$ exposed surface topography

c) $\left[\left(12 \times 10^{-10}\right) \mathrm{m}^{3}\right]<$ Medium erosion $\leq\left[\left(26 \times 10^{-10}\right) \mathrm{m}^{3}\right]+$ exposed surface topography

d) Severe erosion $>\left[\left(26 \times 10^{-10}\right) \mathrm{m}^{3}\right]+$ exposed surface topography
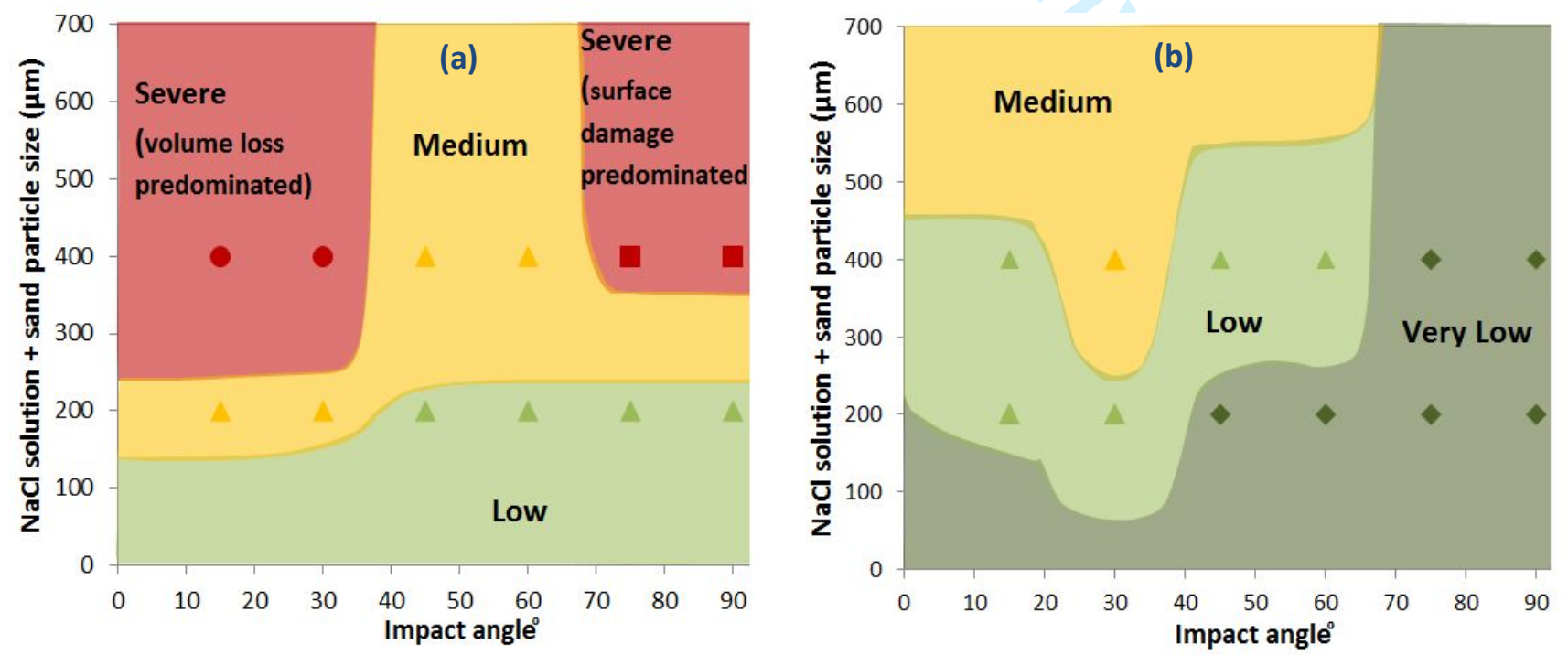
Figure 7: Erosion mode maps in sand + salt solution, a) uncoated composite, and b) coated composite.

As shown in Fig. 7 (a), for the uncoated specimens severe erosive wear is predominating at lower angles of attack because of the cutting action of the erodent. The severity of the erosion increases with an increase in particle size, which results in the volume. On the other hand, with an increase in impact angle, the wear mode transition occurs from severe to medium wear, which again reverts to severe wear. It should be noted that damage of the exposed surface due to material displacement, and plastic deformation, without any change in weight or volume, can also be incorporated into the wear mapping description and is addressed further below [38].

The medium wear area on this map describes a mixture of low and severe wear as a result of volume loss and surface damage. Both the severe and medium wear zones on this map may be regarded as unsafe operating conditions [39]. Moreover, the combined effects of salt solution and sand particles on the uncoated composite result in increasingly severe erosion by removal of material through the cutting action of the erodent at lower angles of attack and impact action of solid particles at higher angles of attack Fig. 5 (a, b, c, d) and Fig. $6(a, b, c, d),[7]$.

By contrast, the coating shows entirely different erosive wear modes than that of uncoated composite Fig. 7 (b). The applications of the coatings have expanded the low wear area of the map of this tribo-system and results in the creation of a new wear regime, i.e. very low wear. Moreover, the severe wear mode has been eliminated from the map. The very low and low wear regimes areas can be regarded as safe operating conditions [39]. Comparison of these maps shows that the coating has increased the erosion resistance of the substrate by a factor of $3[39-41,42-44]$.

\subsection{Erosion mechanism maps}

Erosion mechanism maps show the composite substrate and coating underwent degradation with changes in impact angles and sand and salt water slurries in this tribosystem Fig. 8, [39-41, 42 - 44]. These maps can be helpful to correlate the erosion of the rotor blade with laboratory simulated marine conditions to the actual tidal blade 
operating conditions. The erosion mechanism boundaries are identified on these maps. Moreover, these maps clearly show the transitions in erosion mechanisms with the operating conditions, which depend on the erosion rate and exposed surface morphologies [45 - 46]. It has been well established in the literature that the erosion mechanisms depend on the impact angle $[32,48]$. The theory of erosion presented by Bitter is based on the assumption that up to $<10^{\circ}$ impact angles, the erosion is controlled by the cutting process of the erodent. At $90^{\circ}$, erosion is controlled by the deformation induced by the impact action of the erodent. For the remaining impact angles between $10-90^{\circ}$ erosive wear is progressed by the combined cutting and deformation wear of the erodent [32 - 48]. This theory is not universally accepted as the shape and hardness of the erodent play an important role in the erosion of materials as well [31]. There is maximum loss of the kinetic energy of the erodent at $90^{\circ}$ angle of impact [52].

The empirical erosion mechanism maps for the specimens of the substrate and erosion resistant coating are shown below in Fig. 8.
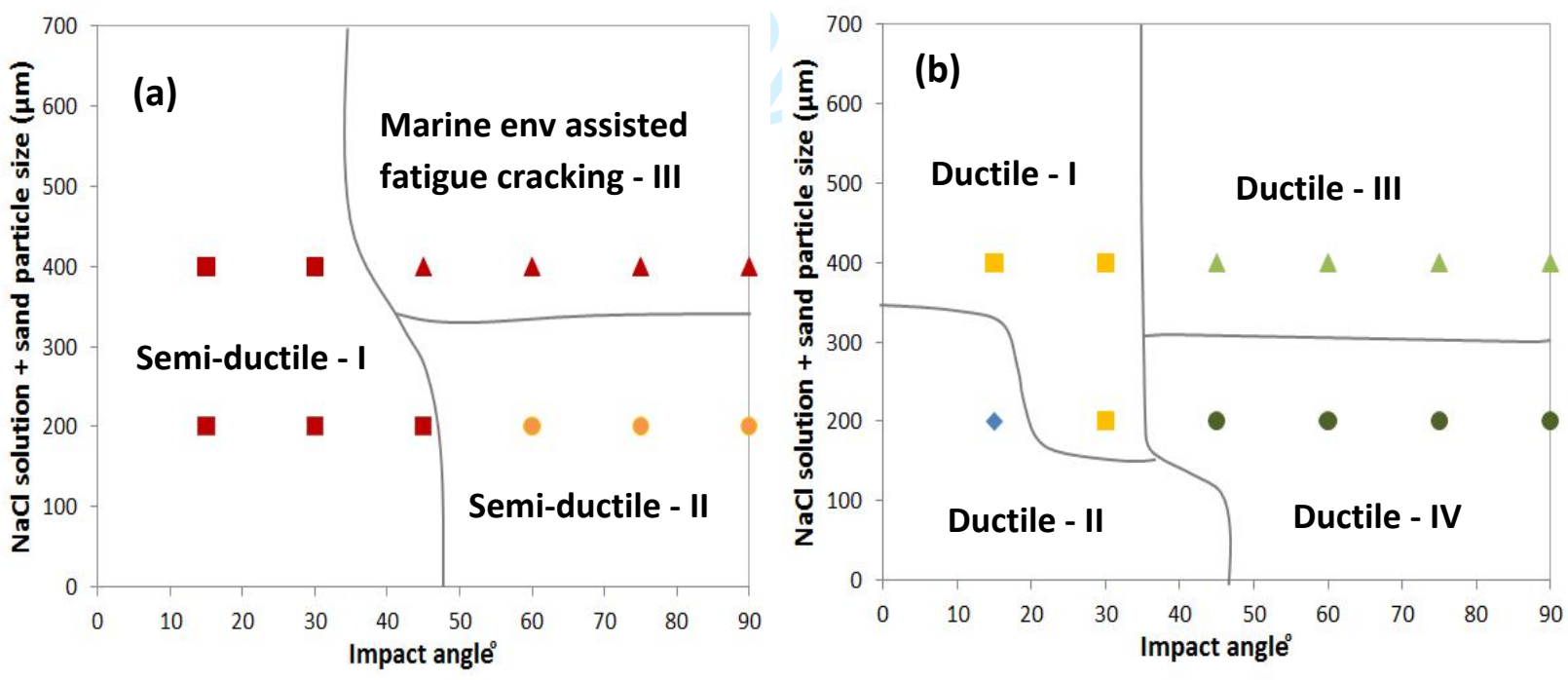

Figure 8: Erosion mechanism maps in sand + salt solution, a) uncoated composite, and b) coated composite.

Erosion regime I - The uncoated composite indicates semi-ductile erosive behaviour in salt water + sand slurries for various impact angles Fig. 4 and Fig. 8 (a). In this region of the erosion mechanistic map, severe degradation of the substrate occurred as a result of higher volume loss Fig. 4 (a, b). The SEM images show the wear progressed by cutting action of the matrix, pit formation, exposure of the fibre and de-bonding at the matrix-reinforcement 
interface in this regime Fig. 5 (a, b) and Fig. $6(a, b)$. An antagonistic erosive effect of salt water and the sand particle is in evidence, which results in an enhanced volume loss of the specimens [7]. The continuous cutting action of the erodent leads to damage of the exposed surface by pit propagation. On the other hand, the erosion resistant polymeric coating exhibits ductile erosion behaviour Fig. 4. The volume loss increases with the increase in solid particle size. The highest volume loss of the coating is still less than that of the uncoated specimens Fig $4(\mathrm{a}, \mathrm{b})$. The mechanisms of erosion are similar for both the coated and uncoated composite, i.e. cutting action of the erodent dominates in this regime Fig. $5(a, b)$ and Fig. 6 (a, b), [7, 52].

Erosion regime II - This region of the mechanism maps is associated with the erosion of the exposed surfaces with smaller particles at higher impact angles for the substrate and lower impact angles for the coating. For the substrate, the exposed surface damage (fibres and matrix) by the impact of the erodent dominates for these angles of attack Fig. 5 (c, d). On the other hand, the micro-cutting process of the specimens dominates in this area for the coatings Fig. $5(e, f)$. This regime relates to the mild erosive wear mode. Both the uncoated and coated composites show ductile erosive wear properties Fig.4 (a).

Erosion regime III - This area of the erosion mechanistic maps is related to the larger size of the sand particle and higher angles of attack. SEM images of the eroded surfaces of both materials show distinctive features Fig. 6 . For the uncoated exposed surface, the erosion is facilitated by an environment-assisted effect, which leads to severe damage of the substrate. Different types of surface degradation are observed, such as de-bonding of the reinforcement fibre-matrix at the interface, fractured fibres, delamination, pit formation, and flake like topographies Fig. 6 (c-d) \& Fig. 8 (a). These observations are similar to those found in the literature for the erosion of the composites [32, 48, 49].

By contrast, the erosion of the coating indicates very little degradation. Tiny indentation/deformation marks of the sand particle are visible on exposed surfaces Figs. 6 (e - h) \& Fig. 8 (b). The coatings act as a ductile erosion material Fig. 4. It has been established in the literature that the impact of the erodent could result in the decrease of mechanical properties (strength) of the fibre reinforced polymer composites up to $40 \%$ [49]. The 
generation of defects in the matrix of the composites presents further issues such as voids, and pores, which affect the mechanical properties of these materials [50].

Erosion regime IV - In this regime of the erosion mechanistic maps, erosion mechanisms are similar to those in the regime III Fig 8. The impact of small solid particle results in less severe damage on the exposed surface of the coatings and there is no indication of surface damage Fig. 5 (g, h).

The above findings have shown that the G10 epoxy glass laminate composite has several tribological issues for tidal turbine applications despite its favourable mechanical properties. As a part of the on-going research for suitable materials for exposure, an erosion resistant polymeric coating was tested in the tidal turbine laboratory simulated environment. This coating has successfully protected the composite substrate in the test conditions above. The coating remained insoluble as well as non-reactive with saltwater $[18,53,54]$. Despite the observed increase in performance, there are however some erosion related issues remaining with this coating in these conditions, which need to be addressed in order to enhance the tribological resistance of such devices to reduce the drag forces on the rotor blades. Erosion maps showing mechanistic changes for both coated and uncoated materials provide a means of optimizing the exposure conditions for both coated and uncoated materials. Future work will be to evaluate composite hybrid coatings for such marine devices.

\section{Conclusions}

1. In this study of erosion of candidate tidal turbine blades, with impact of sand particles in marine simulated conditions, it has been shown that the use of glass fibre reinforced polymer matrix composites presents some challenges due to poor erosion resistance.

2. The application of a polymer based coating has reduced the mass loss and surface degradation of the substrate, during erosion tests at different impact angles and with impact at various sand particle sizes. 
3. For the polymer based coatings, on the erosion maps generated, the low erosive wear regime was expanded to a higher range of impact angles and sand particles. As a result, a new erosive wear mode region, i.e. very low wear, was identified on the map

4. The G10 epoxy glass laminate composite has demonstrated relatively poor erosion resistance despite having favorable mechanical properties, i.e. high stiffness, and strength. However, its relatively high strength can be used to support an erosion resistant polymeric coating, to make it a suitable choice for tidal turbine blade applications.

\section{Acknowledgements}

The authors would like to acknowledge the support of the Interreg (Northern Ireland Ireland - Scotland) Special EU Programmes Grant No SPIRE2_INT - VA - 049 "Storage Platform for the Integration of Renewable Energy (SPIRE 2)" and Belzona Polymerics Limited for coating test samples.

\section{References}

[1] L. Martin, H. Will, S. Aleksandar, H. Adrian, B. Colin, Tidal Energy: Opportunities and Challenges for Renewable Power Generation, International Conference on Engineering Design, ICED' 09 24-27 August 2009, Stanford University, Stanford, CA, USA.

[2]. D. M. Grogan, S. B. Leen, C. R. Kennedy, and C. M. Ó Brádaigh, "Design of composite tidal turbine blades," Renew. Energy, vol. 57, pp. 151-162, 2013.

[3] N. McEwen, R. Evans, and M. Meunier, Cost-Effective Tidal Turbine Blades, 4th International Conference on Ocean Energy, 17 October, Dublin.

[4] https://www.materialstoday.com/composite-applications/features/tidal-turbines-harness-thepower-of-the-sea/.

[5] J. Sloan, Tidal turbine blade toughened for turbulent salt sea, Case study post, 30-11-2012, https://www.compositesworld.com/articles/tidal-turbine-blade-toughened-for-turbulent-salt-sea.

[6] J. N. Goundar and M. R. Ahmed, "Design of a horizontal axis tidal current turbine," Appl. Energy, vol. 111, pp. 161-174, Nov. 2013.

[7] G. Rasool, Shayan Sharifi, Cameron Johnstone, and Margaret M. Stack, Mapping synergy of erosion mechanisms of tidal turbine composite materials in seawater conditions, Bio-TriboCorrosion, DOI: 10.1007/s40735-016-0040-5, June 2016, 2:13. 
[8] J. Zahavi and G. F. Schmitt, "Solid particle erosion of reinforced composite materials," Wear, vol. 71, no. 2, pp. 179-190, Sep. 1981.

[9] G. Huang, Behaviors of glass fiber/unsaturated polyester composites under seawater environment, Mater. Des., 30 (2009), pp. 1337-1340.

[10] S. Sharifi, Cameron Johnstone, Margaret Stack, Tribological Challenges of Scaling Up Tidal Turbine Blades, $11^{\text {th }}$ European Wave and Tidal Energy Conference (EWTEC), September 2015 at Nantes France.

[11] D. Mille, J. F. Mandell, D. D. Samborsky, Bernadette A. Hernandez-Sanchez, and D. Todd Griffith, Performance of Composite Materials Subjected to Salt Water Environments, 2012 AIAA SDM Wind Energy Session.

[12] B. Wei, Hailin Cao and Shenhua, Degradation of basalt and glass fibre/epoxy resin composites in seawater, Corrosion Science, Vol. 53, January 2011, PP. 426-431.

[13] A. Sareen, C. Sapre, M. Selig, Effects of leading edge erosion on wind turbine blade performance, Research Article, Published online 12 July 2013, DOI: 10.1002/we.1649.

[14] M. D. Reder, E. Gongzalez, J. J. Melero, Wind turbine failures - Tackling current problems in failure data analysis, Journal of Physics: Conference Series 753 (2016) 072027.

[15] N. Gaudern, A practical study of the aerodynamic impact of wind turbine blade leading edge erosion, 2014 J. Phys.: Conf. Ser. 524012031.

[16] J. F. Manwell, J. G. Mcgowan, A. L. Rogers, Wind energy explained: theory, design and application, Wiltshire, John Wiley \& Sons Ltd., 2009.

[17] P. Davies, Yapa D. S. Rajapakse, Durability of Composite in Marine Environment, Springer, Vol. 208.

[18] http://www.belzona.com/en/products/1000/1331.aspx. (Product specification sheet BELZONA 1331, FN10027).

[19] A. Abouel-Kasem, Y. M. Abd-elrhman, K. M. Emara, and S. M. Ahmed, "Design and Performance of Slurry Erosion Tester," J. Tribol., vol. 132, no. 2, p. 021601, Apr. 2010.

[20] G. R. Desale, B. K. Gandhi, and S. C. Jain, "Improvement in the design of a pot tester to simulate erosion wear due to solid-liquid mixture," Wear, vol. 259, no. 1-6, pp. 196-202, Jul. 2005. 
[21] A. A. Gadhikar, A. Sharma, D. B. Goel, and C. P. Sharma, "Fabrication and Testing of Slurry Pot Erosion Tester," Trans. Indian Inst. Met., vol. 64, no. 4-5, pp. 493-500, Dec. 2011.

[22] W. Tsai, J. A. C. Humphrey, I. Cornet, and A. V. Levy, "Experimental measurement of accelerated erosion in a slurry pot tester," Wear, vol. 68, no. 3, pp. 289-303, May 1981.

[23] A. Muratoglu, M. Ishak Yuce, Performance Analysis of Hydrokinetic Turbine Blade Sections, Avestia Publishing, Advances in Renewable Energy, Vol. 2, the year 2015.

[24] P. Fraenkel, Practical tidal turbine design considerations: a review of technical alternatives and key design decisions leading to the development of the SeaGen 1.2MW tidal turbine, Proceedings Fluid Machinery Group - Ocean Power Fluid Machinery Seminar Institution of Mechanical Engineers 19 October 2010, London.

[25] K. K.Wong, Hector Mcl. Clark, (1993), A model of particle velocities and trajectories in a slurry pot erosion tester, Wear 160 (1), pp 95-104.

[26] D. Val, Leon Chernin, Reliability of tidal stream turbine blades, Conference Paper, $11^{\text {th }}$ International Conference on Applications of Statistics and Probability in Civil Engineering (ICASP 2011), at Zurich, Switzerland, DOI: 10.1201/b1 1332-270.

[27] H. J. Amarendra, G. P. Chaudhari, and S. K. Nath, "Synergy of cavitation and slurry erosion in the slurry pot tester," Wear vol. 290-291, pp. 25-31, Jun. 2012.

[28] M. Lindgren, J. Perolainen, Slurry pot investigation of influence of erodent characteristics on the erosion resistance of austenitic and duplex stainless steel grades, Wear, Vol. 319, No. 1-2, (2014), pp. 38-48.

[29] B. N-M, Karger-Kocsis J., Effects of fibre content and relative fibre orientation on the solid particle erosion of GF/PP composites, Wear 252, (2002), Pages 80-87.

[30] T. Sinmazcelik, Isa Taskiran, Erosive wear behaviour of polyphenylenesulphide (PPS) composites, Materials and Design 28 (2007) 2471-2477.

[31] I. Finnie, J. Wolak, and Y. Kabil, Erosion of metals by solid particles, Journal of Materials, Vol. 2, (1967), pp 682-700.

[32] J. W. M. Mens and A.W.J. de Gee, Erosion in seawater slurries, Tribology International, Vol. 19, issue 2, April 1986, PP. 59-64. 
[33] B. D. Jana, M. M. Stack, A note on threshold velocity criteria for modelling the solid particle erosion of WC/Co MMCs, Wear, Vol. 270, No. 7-8 (2011), pp. 439-445.

[34] G. Rasool, M.M. Stack, Mapping the role of $\mathrm{Cr}$ content in dry sliding of steels: Comparison between maps for material and counterface, Tribology International 80(2014) 49-57.

[35] G. Rasool, S. Mridha and M.M. Stack, Mapping wear mechanisms for TiC/Ti composite coatings, Wear, Volumes 328-329, 15 April 2015, Pages 498-508.

[36] G. Rasool and M.M. Stack, Tribo-Oxidation maps for Ti against steel, Journal of Tribology International, Volume 9, November 2015, Pages 258-266.

[37] G. Rasool and M.M. Stack, Wear maps for TiC composite based coatings deposited on 303stainless steel, Tribology International, Volume 74, June 2014, Pages 93-102.

[38] B. Bhushan, Introduction to Tribology, ISBN 0-471-15893-3, Page 331.

[39] S. C. Lim, Recent developments in wear mechanism maps, Tribology International 31 (1998), Pages 87-97.

[40] J. Zhang and A. T. Alpas, Transition between mild and severe wear in aluminium alloys, Acta Mater., 45 (1997), Pages 513-528.

[41] S. Wilson, A. T. Alpas, Wear mechanism maps for metal matrix composites, Wear, 212 (1997), Pages 41-49.

[42] G. Rasool, S. Mridha and M.M. Stack, Mapping wear mechanisms for TiC/Ti composite coatings, Journal of Wear, Volumes 328-329, 15 April 2015, Pages 498-508.

[43] G. Rasool and M.M. Stack, Tribo-Oxidation maps for Ti against steel, Journal of Tribology International, Volume 9, November 2015, Pages 258-266.

[44] G. Rasool and M.M. Stack, Wear maps for TiC composite based coatings deposited on 303stainless steel, Tribology International, Volume 74, June 2014, Pages 93-102.

[45] L. CYH, Lim SC, Lee KS., Wear of TiC-coated carbide tools in dry turning, Wear 1999;225229:354-67.

[46] S. Wilson, Alpas AT., TiN coating wear mechanisms in dry sliding contact against high-speed steel. Surf Coat Technol. 1998; 108-109:369-76. 
[47] H. M. Slot, Corne Rentrop, Edwin Gelinck, Emile Van Der Heide, Leading edge erosion of coated wind turbine blades: Review of coating models, Renewable Energy 80 (2015), pp. 837-848.

[48] J. G. Bitter, A study of erosion phenomena, Wear, Vol. 6, PP 169-190, 1963.

[49] E. Kosa and A. Göksenli, Effect of Impact Angle on Erosive Abrasive Wear of Ductile and Brittle Materials, World Academy of Science, Engineering and Technology International Journal of Mechanical and Mechatronics Engineering Vol:9, No:9, 2015.

[50] Polymer-based Nanocomposites for Energy and Environmental Applications

https://books.google.co.uk/books?isbn=0081019114.

Advance material by design, Chapter3, Polymer Matrix Composite, DIANE Publishing.

[51] G. Fiore, and S. Selig Michael, Simulation of damage progression on wind turbine blades subject to particle erosion, AIAA SciTech, 4-8 January 2016, San Diego, California, $5^{\text {th }}$ AIAA Aerospace Science Meeting.

[52] Y. Fouad, M. El. Meniawi, A. Afifi, Erosion behaviour of epoxy based unidirectional (GFRP) composite materials, Alexandria Engineering Journal, (2011) 50, pp.29-34.

[53] R. A. Dickie and F. Louis Floyd, Polymeric material for corrosion control: An Overview, DOI: 10.1021/bk-1986-0322.ch001.

[54] L. Mardare and L. Benea, Development of anticorrosive polymer nanocomposite coatings for corrosion protection in marine environment, IOP Conference series: Material Science and Engineering, 209 (2017), 012056, DOI: 10. 1088/1757-899X/209/1/012056. 